Check for updates

Cite this: DOI: 10.1039/c8sm01412d

\section{Unravelling the structural rearrangement of polymer colloidal crystals under dry sintering conditions $\dagger$}

\author{
Alexey V. Zozulya, (D)*a Ivan A. Zaluzhnyy, (D) ${ }^{\text {bc }}$ Nastasia Mukharamova, ${ }^{\text {b }}$ \\ Sergey Lazarev, (D) bd Janne-Mieke Meijer, (D) † $^{\mathrm{e}}$ Ruslan P. Kurta, ${ }^{a}$ Anatoly Shabalin, $\S^{\mathrm{b}}$ \\ Michael Sprung, ${ }^{b}$ Andrei V. Petukhov (iD ${ }^{\text {ef }}$ and Ivan A. Vartanyants (DD ${ }^{\text {bc }}$
}

\begin{abstract}
The structural rearrangement of polystyrene colloidal crystals under dry sintering conditions has been revealed by in situ grazing incidence $X$-ray scattering. The measured diffraction patterns were analysed using distorted wave Born approximation (DWBA) theory and the structural parameters of the as-grown colloidal crystals of three different particle sizes were determined for the in-plane and out-of-plane directions in a film. By analysing the temperature evolution of the diffraction peak positions, integrated intensities, and widths, the detailed scenario of the structural rearrangement of crystalline domains at the nanoscale has been revealed, including thermal expansion, particle shape transformation and crystal amorphisation. Based on DWBA analysis, we demonstrate that in the process of dry sintering, the shape of colloidal particles in a crystal transforms from a sphere to a polyhedron. Our results deepen the understanding of the thermal annealing of polymer colloidal crystals as an efficient route for the design of new nano-materials.
\end{abstract}

Received 10th July 2018 Accepted 27th July 2018

DOI: $10.1039 / c 8 s m 01412 d$

rsc.li/soft-matter-journal

\section{Introduction}

Photonic crystals are optical structures with periodic modulations of medium dielectric susceptibility leading to the appearance of photonic band gaps. ${ }^{1}$ Due to their optical properties of confining and controlling electromagnetic waves in three dimensions, photonic crystals have found various applications in optoelectronics, ${ }^{2}$ acousto-optics, ${ }^{3}$ photovoltaics ${ }^{4}$ and thermoelectrics. ${ }^{5}$ Photonic crystals can be fabricated in a highly controllable fashion by nanolithography methods, ${ }^{6,7}$ which involve many-step fabrication

\footnotetext{
${ }^{a}$ European XFEL GmbH, Holzkoppel 4, Schenefeld D-22869, Germany. E-mail: alexey.zozulya@xfel.eu

${ }^{b}$ Deutsches Elektronen-Synchrotron DESY, Notkestraße 85, Hamburg D-22607, Germany

${ }^{c}$ National Research Nuclear University MEPhI (Moscow Engineering Physics Institute), Kashirskoye ch. 31, Moscow 115409, Russia

${ }^{d}$ National Research Tomsk Polytechnic University (TPU), Lenin Avenue 30, Tomsk 634050, Russia

${ }^{e}$ Van't Hoff Laboratory for Physical and Colloid Chemistry, Department of Chemistry and Debye Institute for Nanomaterials Science, Utrecht University, Padualaan 8, $3584 \mathrm{CH}$, The Netherlands

${ }^{f}$ Laboratory of Physical Chemistry, Department of Chemical Engineering and Chemistry and Institute for Complex Molecular Systems, Eindhoven University of Technology, P.O. Box 513, 5600 MB, Eindhoven, The Netherlands

$\dagger$ Electronic supplementary information (ESI) available. See DOI: 10.1039/c8sm01412d \$ Present address: Department of Physics, University of Konstanz, D-78457 Konstanz, Germany.

$\S$ Present address: University of California, 9500 Gilman Dr., La Jolla, San Diego, California 92093, USA.
}

procedures and are quite expensive. The self-assembly of colloidal particles into 3D crystals provides an alternative fabrication technique to nanolithography, with the extended possibilities of tailoring the optical properties of a photonic crystal by changing the particle size. ${ }^{8}$ In this way, colloidal crystals can be synthesized from a colloidal suspension using convective assembly methods under ambient conditions, ${ }^{9,10}$ representing a cost-efficient and tunable fabrication technique.

The shape of the colloidal particles is responsible for the self-assembly of colloidal crystals, ${ }^{11-14}$ and it directly affects their dimensionality, ${ }^{15}$ phase behaviour ${ }^{16,17}$ and optical properties. ${ }^{18}$ Thermal or chemical annealing can be used to modify the shape of self-assembled colloidal particles and, hence, tailor the photonic band gap properties of a colloidal crystal. ${ }^{19-23}$ As a result of the annealing treatment, the spherical shape of a colloidal particle changes to a faceted shape, which provides a new route for the fabrication of polyhedral particles. ${ }^{24}$ Therefore, the pathway of particle shape transformation under thermal or chemical annealing conditions is an important aspect in structural studies of colloids, including potential applications of polyhedral colloidal particles for the synthesis of reconfigurable colloidal materials ${ }^{25}$ and in catalysis research. $^{26,27}$

Different methods can be employed to characterise colloidal systems depending on the size of the particles. Optical and confocal laser scanning microscopies can be efficiently applied to visualize $3 \mathrm{D}$ colloidal systems, ${ }^{28}$ albeit the resolution of optical methods is typically set by the Abbe diffraction limit 
and particles smaller than $500 \mathrm{~nm}$ are difficult to resolve. Scanning electron microscopy (SEM) and transmission electron microscopy (TEM) provide sub-nanometer resolution. ${ }^{29}$ However, due to the low penetration depth of electrons, SEM and TEM methods can probe mostly the surface morphology of a specimen at a limited field of view. X-ray scattering methods, being free from these limitations due to the high penetration depth, enable nondestructive access to the interior of colloidal crystals at nanometer resolution and below. Furthermore, X-ray methods can probe sample areas ranging from hundreds of microns down to tens of nanometers, thus providing both mesoscopic and nanoscale structural information. Finally, X-ray techniques are well suited for in situ measurements involving complex sample environments (e.g. temperature, ${ }^{30}$ pressure, ${ }^{31}$ and chemical conditions ${ }^{32}$ ).

Synchrotron X-ray scattering has been extensively applied for structural studies of colloidal crystals at ambient conditions. ${ }^{33-40}$ The rearrangement of polymer colloidal assemblies under thermal annealing conditions has been studied by in situ small angle X-ray scattering (SAXS). ${ }^{41-44}$ Recently, the details of the structural transitions in polystyrene (PS) colloidal crystals upon heating treatment, including particle faceting and crystal melting, have been revealed. ${ }^{30,45}$ In the present work, we employ an in situ grazing incidence X-ray scattering technique to unravel the details of the structural evolution of PS colloidal crystal domains under dry sintering conditions. As compared to standard transmission SAXS geometry, grazing incidence X-ray scattering is sensitive to both the in-plane and out-of-plane structural order, and furthermore, it provides better control during thermal annealing due to the whole sample area forming a thermal contact.

\section{Methods}

\subsection{Experiment}

The schematics and details of the experimental setup are presented in Fig. 1. X-ray scattering experiments were performed at the P10 coherence beamline of the PETRA III synchrotron source at DESY, Hamburg. The incident X-ray beam with a photon energy of $7.74 \mathrm{keV}$ was adjusted to $50 \times 50 \mu \mathrm{m}^{2}$ size using beam-defining slits. The sample was aligned vertically on a 5 -axis positioning stage such that the scattering plane was horizontal. Diffraction patterns were acquired using a 2D detector Pilatus $300 \mathrm{~K}$ with an area of $487 \times 619$ pixels and a pixel size of $172 \mu \mathrm{m}$. The detector was positioned at $5.1 \mathrm{~m}$ downstream from the sample. The incident angle $\alpha_{i}$ for the studied samples was adjusted to $0.5^{\circ}$, which allowed the X-ray scattering signal to be optimised without obscuring effects from the sample holder. The scattering patterns were acquired by collecting 100 detector frames with an exposure time of $1 \mathrm{~s}$ each. Our measurements benefited from the high incident flux of the synchrotron X-ray beam of $1.1 \times 10^{11}$ photons per s/50 $\times 50 \mu \mathrm{m}^{2}$ and the fact that colloidal crystals were grown on thin glass coverslips of $170 \mu \mathrm{m}$ thickness, providing a relatively high transmission of $22 \%$ at normal incidence for the used photon energy. Using grazing incidence geometry lowers the transmission of the samples significantly, resulting in values of the order of $10^{-5}$. The air scattering background was eliminated by positioning the

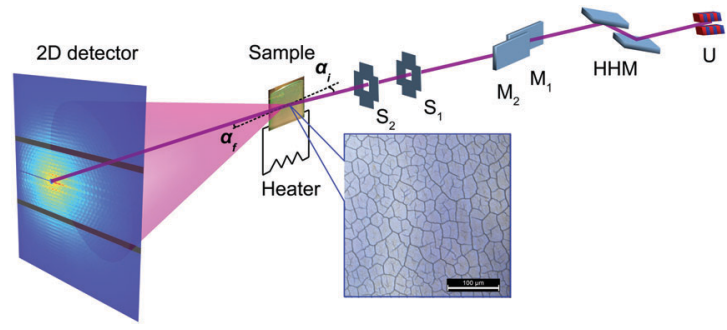

Fig. 1 Schematics of the experimental setup. X-ray beam produced by an undulator $(U)$ is guided through a high-heatload monochromator (HHM) and two $X$-ray mirrors $\left(M_{1}, M_{2}\right)$. The size of the beam impinging on a sample at grazing angle $\alpha_{i}$ was adjusted by the beam-defining slits $S_{1}$ and $\mathrm{S}_{2}$. The sample was mounted on a heater stage inside the vacuum chamber and the scattering signal was acquired using the 2D detector placed downstream from the sample. Inset: Optical micrograph of a PS colloidal crystal sample; scale bar $100 \mu \mathrm{m}$.

sample inside a vacuum chamber and using an evacuated flight tube between the sample and detector. To protect the detector from the transmitted beam and optimize the scattering signal acquisition at high $q$-values, the beamstop (tungsten cylinder of $3 \mathrm{~mm}$ diameter) was positioned inside the flight tube in front of the detector.

\subsection{Sample preparation}

PS colloidal crystal films were fabricated by the vertical deposition method. ${ }^{10}$ Spherical colloidal particles were synthesised by polymerization of an aqueous solution of styrene using potassium persulphate as the initiator. Initial colloidal suspensions for the studied samples with particle diameters of 420, 272 and $194 \mathrm{~nm}$ (referred to further as samples A, B and C, respectively) contained $0.8,0.25$ and $0.5 \%$ volume fractions of colloidal particles in water. The particle polydispersity values measured by dynamic light scattering (DLS) were 2.1, 4 and 3.5\% (see Table 1). Thin glass substrates were immersed into colloidal suspensions and subsequently dried at $50{ }^{\circ} \mathrm{C}$ for several days under ambient conditions. The fabricated colloidal crystal films consisted of 20-80 monolayers of PS spherical particles and typically exhibited a cracked texture composed of domains with an average size of 10-50 $\mu \mathrm{m}$ (typical optical microscopy image is shown as an inset in Fig. 1). The colloidal crystal samples were mounted onto the sample holder using silver paste to ensure a good thermal contact. The copper

Table 1 Structural parameters of PS colloidal crystal samples at RT

\begin{tabular}{llll}
\hline Sample & $\mathrm{A}$ & $\mathrm{B}$ & $\mathrm{C}$ \\
\hline$D_{\mathrm{DLS}}, \mathrm{nm}$ & 420 & 272 & 194 \\
$D_{\mathrm{GTSAXS}}, \mathrm{nm}$ & 419 & 257 & 197 \\
Polydispersity (DLS), \% & 2.1 & 4 & 3.5 \\
$c / a$ ratio & 0.99 & 1.00 & 0.98 \\
Packing & rhcp & rhcp & rhcp \\
hcp fraction & 0.6 & 0.2 & 0.8 \\
fcc fraction & 0.4 & 0.8 & 0.2 \\
Number of layers & 30 & 70 & 60 \\
Thickness, $\mu \mathrm{m}$ & 10.3 & 14.7 & 9.7 \\
Lateral domain size, $\mu \mathrm{m}$ & 4.2 & 2.3 & 3.7 \\
In-plane $\Delta d / d, \%$ & 4.3 & 5.4 & 4.8 \\
Out-of-plane $\Delta d / d, \%$ & 5.1 & 6.8 & 3.5 \\
In-plane mosaicity, mrad & 51 & 68 & 35 \\
Out-of-plane mosaicity, mrad & 43 & 54 & 48
\end{tabular}


block of the sample holder was integrated into the vacuum chamber. Heating was supplied by two parallel connected heating elements integrated into the copper block, and the temperature was measured using two PT100 sensors embedded into the sample holder. Temperature and heating power were controlled by using a LakeShore 340 temperature controller. During the measurements, the temperature of the sample was raised incrementally starting from room temperature (RT). After each temperature increment, a waiting time of 5 min was applied before collecting the data to reach thermal equilibrium within the sample.

\section{Results and discussion}

\subsection{GTSAXS simulations}

To elucidate the discussion of the results, we first consider the geometry of the X-ray scattering experiment under grazing incidence conditions. In a conventional grazing incidence small angle X-ray scattering (GISAXS) geometry, ${ }^{46,47}$ the incident beam with a wave vector $\mathbf{k}_{\mathrm{i}}$ illuminates the sample surface at a grazing angle $\alpha_{\mathrm{i}}$, typically exceeding the critical angle of the total external reflection $\alpha_{c}$ of the substrate material, and it generates a scattered beam with a wave vector $\mathbf{k}_{\mathrm{f}}$ exiting the surface at an angle $\alpha_{\mathrm{f}}$. The direction of the scattered wave vector is defined by two angles, $2 \theta_{\mathrm{f}}$ and $\alpha_{\mathrm{f}}$, for the in-plane and out-of-plane directions (Fig. 2). The components of the wave vector transfer $\mathbf{q}=\mathbf{k}_{\mathrm{f}}-\mathbf{k}_{\mathrm{i}}$ are related to the angular coordinates:

$$
\begin{gathered}
q_{x}=k_{0}\left[\cos \left(2 \theta_{\mathrm{f}}\right) \cos \left(\alpha_{\mathrm{f}}\right)-\cos \left(\alpha_{\mathrm{i}}\right)\right], \\
q_{y}=k_{0} \sin \left(2 \theta_{\mathrm{f}}\right) \cos \left(\alpha_{\mathrm{f}}\right), \\
q_{z}=k_{0}\left[\sin \left(\alpha_{\mathrm{f}}\right)+\sin \left(\alpha_{\mathrm{i}}\right)\right] .
\end{gathered}
$$

For the colloidal crystals studied in this work, the specularly reflected signal was hampered by the surface roughness, which prevented us from using GISAXS geometry. Instead, we employed the grazing incidence transmission small angle X-ray scattering (GTSAXS) geometry, ${ }^{48,49}$ where only the forward transmitted

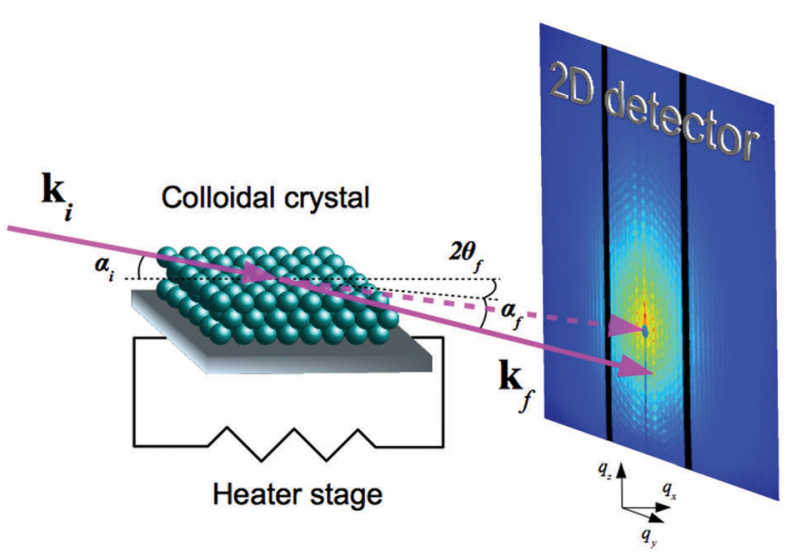

Fig. 2 Scattering geometry of the GTSAXS experiment. The incident beam with a wave vector $\mathbf{k}_{\mathbf{i}}$ enters the sample surface at a grazing angle $\alpha_{i}$. The scattered beam with a wave vector $\mathbf{k}_{\mathrm{f}}$ is defined by the in-plane and out-of-plane scattering angles $2 \theta_{\mathrm{f}}$ and $\alpha_{\mathrm{f}}$, respectively. part of a scattering pattern below the sample horizon $\left(\alpha_{\mathrm{f}}<0\right)$ is analysed, as illustrated in Fig. 2.

In order to determine the structural parameters of colloidal samples, the GTSAXS data were analysed by X-ray scattering simulations in the frame of DWBA theory. ${ }^{50-54}$ In the case of colloidal particles supported on a substrate, DWBA considers the scattering amplitudes in a particle and at an interface between vacuum and substrate, as described by the Fresnel reflection and transmission coefficients. According to DWBA theory, in total, four scattering amplitudes contribute to the resulting scattering signal from a single particle: (1) the amplitude of kinematical scattering in a particle described by the Born approximation, (2) the reflection from a substrate followed by the scattering event in a particle, (3) the scattering in a particle followed by the reflection from a substrate and (4) the reflection from a substrate followed by the scattering event and subsequent reflection from the substrate. In the GTSAXS geometry, the scattering signal below the sample horizon is considered, ${ }^{48}$ and hence only the first two scattering channels contribute to the resulting scattering amplitude of a single particle, $A_{\mathrm{p}}(\mathbf{q})=A_{1}(\mathbf{q})+A_{2}(\mathbf{q}):$

$$
\begin{gathered}
A_{1}(\mathbf{q})=\chi_{0}\left(k^{2} / 4 \pi\right) \int S(\mathbf{r}) \mathrm{e}^{-i\left(\mathbf{q}_{\|}+\mathbf{q}_{z, 1}\right) \mathbf{r}} \mathrm{d} \mathbf{r}, \\
A_{2}(\mathbf{q})=\chi_{0}\left(k^{2} / 4 \pi\right) R\left(\alpha_{i}\right) \int S(\mathbf{r}) \mathrm{e}^{-i\left(\mathbf{q}_{\|}+\mathbf{q}_{z, 2}\right) \mathbf{r}} \mathrm{d} \mathbf{r},
\end{gathered}
$$

where $\mathbf{q}_{\|}=\left(q_{x}, q_{y}\right)$ represents the in-plane components and $q_{z, 1}=-k_{z}^{\mathrm{f}}-k_{z}^{\mathrm{i}}$ and $q_{z, 2}=-k_{z}^{\mathrm{f}}+k_{z}^{\mathrm{i}}$ are the out-of-plane components of the wave vector transfer. $k=2 \pi / \lambda$ is the wave vector in a vacuum and $\chi_{0}$ is the 0 -th order Fourier component of susceptibility of the particle material at a given X-ray wavelength $\lambda$. The shape function $S(\mathbf{r})$ is equal to unity inside a particle and it is zero outside. $R\left(\alpha_{\mathrm{i}}\right)=\left(k_{z}^{\mathrm{i}}-\tilde{k}_{z}^{\mathrm{i}}\right) /\left(k_{z}^{\mathrm{i}}+\tilde{k}_{z}^{\mathrm{i}}\right)$ is the Fresnel reflection coefficient for the incident wave. $k_{z}$ and $\tilde{k}_{z}$ are $z$-components of the wave vector in a vacuum and substrate media, respectively. The total scattering amplitude $A_{\text {cr }}(\mathbf{q})$ from a colloidal crystal lattice consisting of $N$ monodisperse particles is the sum of the scattering amplitudes from each particle with a radius-vector $r_{\mathrm{i}}$ :

$$
A_{\text {cr }}(\mathbf{q})=A_{\mathrm{p}}(\mathbf{q}) \sum_{i=1}^{N} \mathrm{e}^{-i \mathbf{q} \mathbf{r}_{\mathrm{i}}} .
$$

The GTSAXS intensity distribution has been calculated as $I_{\text {GTSAXS }}(\mathbf{q})=\left|A_{\text {cr }}(\mathbf{q})\right|^{2}$.

\subsection{GTSAXS analysis}

GTSAXS patterns measured at RT for the three colloidal crystals A, B and C, consisting of PS spherical particles of 420, 272 and $194 \mathrm{~nm}$ in diameter, respectively, are shown in Fig. 3a-c. Scattering patterns are displayed for the reciprocal space areas covering the same number of diffraction peaks, which correspond to $q$-ranges of $0.025 \times 0.025,0.041 \times 0.041$ and $0.050 \times 0.050 \AA^{-2}$ for the samples A, B and C, respectively. The achieved resolution in reciprocal space of $1.32 \times 10^{-4} \AA^{-1}$ was defined by the pixel size of the $2 \mathrm{D}$ detector and the sample-to-detector distance at $7.74 \mathrm{keV}$ X-ray energy (see Methods section for details). Indexed sets of 

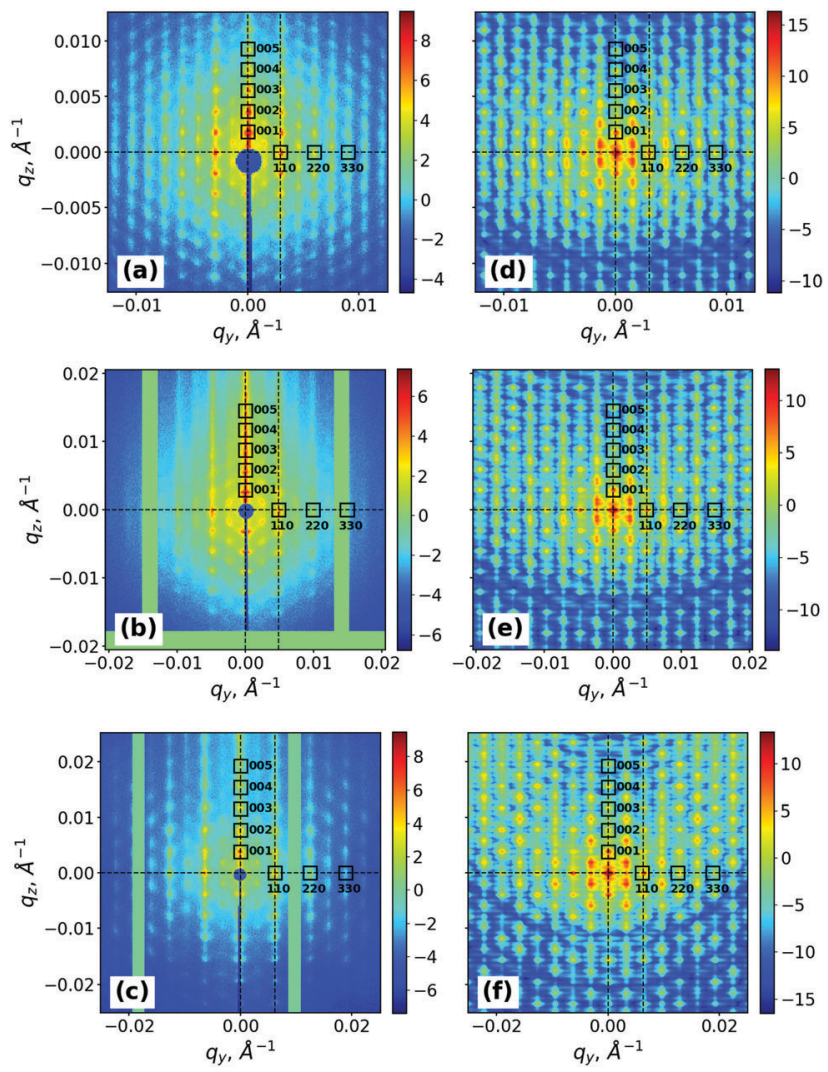

Fig. $3(a-c)$ Experimental and $(d-f)$ simulated GTSAXS patterns for the three PS colloidal crystals ( $a, d) A$, (b, e) B, and (c, f) C at RT. Indexed sets of in-plane and out-of-plane reflections are marked by rectangles. Dashed lines indicate the directions of the $q_{y^{-}}$and $q_{z}$-profiles used for the analysis; the vertical line across the $(-1-10)$ peak is not shown for better data visibility. A circular area around the direct beam was covered by the beamstop, and the vertical stripes of missing data are due to the gaps between detector tiles; the horizontal stripe in (b) was not captured by the detector. Intensities are represented in logarithmic scale.

in-plane reflections of (110)-type and out-of-plane reflections of (001)-type employed for the analysis are depicted by rectangles. Dashed lines denote the $q_{y^{-}}$and $q_{z}$-directions used to extract intensity profiles for fitting analysis. In the case of the GTSAXS geometry, the scattering pattern is dominated by the kinematical scattering contribution and less influenced by the re-scattering channels inherent to conventional GISAXS measurement. In particular, the characteristic GISAXS features such as specular and Yoneda peaks are not present in the GTSAXS data. For all the measured samples, the central and side peaks along the $q_{z}$-direction reveal strong truncation rods that originate from the substrate scattering under grazing incidence angles. Due to variations of particle polydispersity and defect concentration, we observed a different number of diffraction orders for the studied colloidal crystals. For sample A, diffraction peaks up to the 13th order were observed, indicating a high structural quality. Meanwhile, for sample B, only 5 diffraction orders were observed, indicating the rather low quality of the sample, which can be related to the high polydispersity of $4 \%$ (see Table 1). Sample C revealed 7 diffraction orders, indicating an intermediate degree of long-range ordering.
To obtain quantitative sample characteristics, GTSAXS simulations using eqn (1)-(6) were implemented as in-house developed Python scripts. Simulated patterns were calculated on a $1600 \times 1600 \mathrm{px}^{2}$ grid for the $q$-ranges corresponding to the GTSAXS data from three PS colloidal crystals (Fig. 3d-f). To simulate the total scattering amplitude from a colloidal crystal, we first calculated the scattering amplitude from a single particle according to DWBA theory, which was then summed over the particles constituting a single layer and, lastly, the summation over a stack of particle layers was performed. The stacking of $N_{1}$ particle layers in a random hexagonal closepacked (rhcp) arrangement ${ }^{55}$ was implemented by introducing $n_{\text {hcp }}$ hexagonal close-packed (hcp) layers (layer sequence ABAB...) and $\left(N_{1}-n_{\text {hcp }}\right)$ layers of face-centered cubic (fcc) structure (layer sequence ABCABC. . .). Peak broadening caused by lattice spacing variation was taken into account by a convolution of the simulated data with a Voigt function, which also included the instrumental broadening due to the incident beam divergence of $4 \times 28 \operatorname{\mu rad}^{2}$ (vertical $\times$ horizontal). ${ }^{30}$

The experimental $q_{y^{-}}$and $q_{z}$-profiles extracted from the GTSAXS patterns along the directions depicted by dashed lines in Fig. 3 were fitted with calculated curves by adjusting the structural parameters such as particle diameter, lateral domain size, number of layers, lattice deformation and mosaicity values. The thus obtained best-fit curves (Fig. S1, ESI $\dagger$ ) yielded the in-plane and out-of-plane structural parameters of the studied colloidal crystals under RT conditions, which are summarised in Table 1. Particle diameter values in the colloidal crystals determined by GTSAXS slightly deviate (within several percent) from the results of DLS measurements on the colloidal suspensions. Furthermore, for the samples A and C, we found that the in-plane lattice spacing $a$ is 1-2\% larger than the out-of-plane spacing $c$. The observed in-plane lattice dilatation might be caused by the rhcp stacking nature of particle layers as well as the surface tension affecting the formation of a colloidal crystal during solvent evaporation. By varying the hcp and fcc fractions in the rhcp stacking, the corresponding values were evaluated within $10 \%$ accuracy. For all three samples, the fitting curves for the in-plane direction (Fig. S1, top panels and Fig. S5, ESI $\dagger$ ) yielded a higher intensity at the $(1 / 21 / 20)$ reciprocal space point, which we relate to the simplified modelling of the rhcp stacking disorder by a linear combination of hep and fcc structures. Using the determined values of particle diameter and the number of layers, the total thickness of a colloidal crystal film can be estimated, which was found to increase from $9.7 \mu \mathrm{m}$ for sample $\mathrm{C}$ up to $14.6 \mu \mathrm{m}$ for sample $\mathrm{B}$, with an intermediate value of $10.3 \mu \mathrm{m}$ for sample A. The determined values of lateral domain size, lattice deformation and mosaicity indicated the highest degree of structural quality of sample A, the lowest quality of sample B and an intermediate degree for sample $\mathrm{C}$.

\subsection{Temperature evolution of GTSAXS peak parameters}

Once the structural parameters of the colloidal crystals were quantified at RT conditions, we analysed the in situ GTSAXS data measured upon incremental heating of the samples in a wide temperature range from RT to $385 \mathrm{~K}$. Fig. 4 shows the 
GTSAXS patterns for samples A, B and C at selected temperatures: $300,355,376,381$ and $385 \mathrm{~K}$. The patterns are displayed for the $q$-range of $0.0643 \times 0.0643 \AA^{-1}$ representing almost the full range of the $2 \mathrm{D}$ detector (square area of $487 \times 487 \mathrm{px}^{2}$ of a Pilatus $300 \mathrm{~K}$ detector, see Methods section). By visual inspection of the diffraction peaks and the diffuse scattering distribution, one can readily identify the intermediate states of a colloidal crystal in the process of dry sintering. In the temperature range from RT to the annealing temperature $T_{\mathrm{a}}=355 \mathrm{~K}$, the peak shapes and positions exhibit no significant changes, while the isotropic diffuse scattering becomes suppressed. With a further increase of temperature up to $T=376 \mathrm{~K}$, the high-order diffraction peaks start to vanish, indicating a gradual loss of long-range ordering in the colloidal crystal. The fading of longrange order is accompanied by a transition from an isotropic to anisotropic diffuse scattering distribution in the form of two inclined flares depicted by white arrows in Fig. 4 at $T=376 \mathrm{~K}$. The anisotropy of the diffuse scattering indicates the onset of particle plastic deformation and shape transformation from a spherical to a faceted shape. Above $T=381 \mathrm{~K}$, the abrupt decay of lower diffraction orders signifies the total loss of ordering and the formation of an amorphous polymer film.

To unravel the behaviour of the colloidal crystals under dry sintering conditions, we analysed the GTSAXS data by evaluating the diffraction peak parameters, such as position, integrated intensity and widths, as a function of temperature. The in-plane

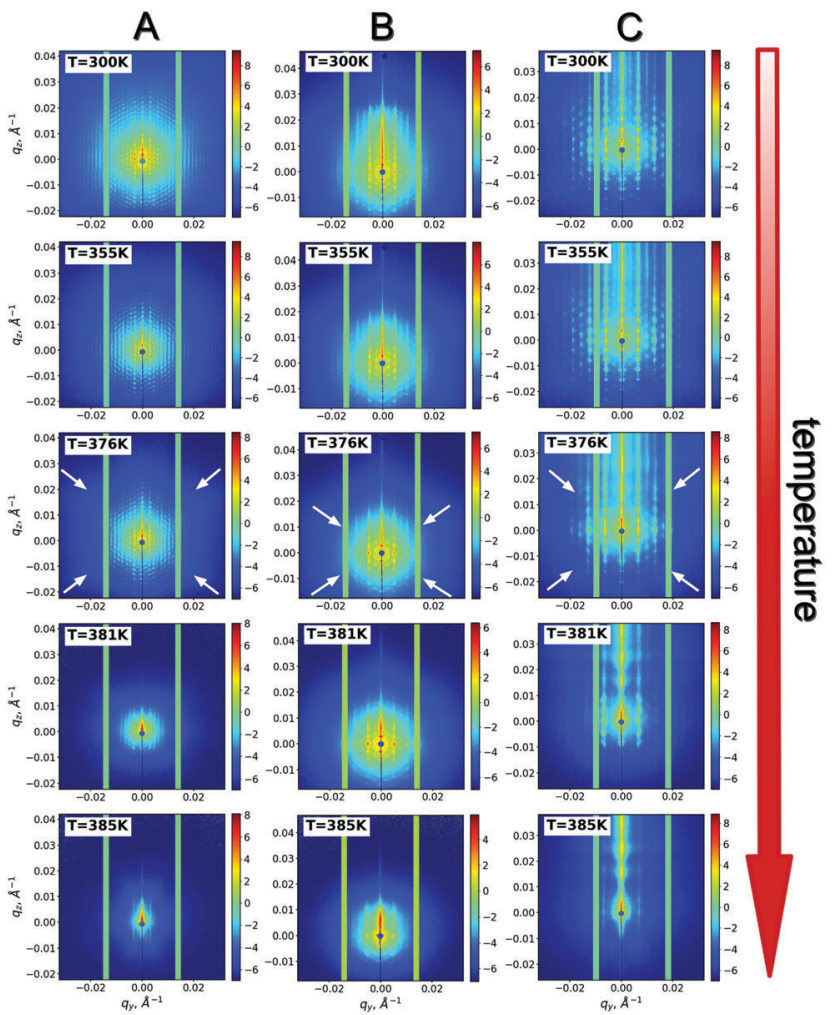

Fig. 4 In situ GTSAXS patterns for the PS colloidal crystal samples A (left column), B (middle column), and C (right column) measured during incremental heating in the range from RT to $T=385 \mathrm{~K}$. Intensities are represented in the logarithmic scale.
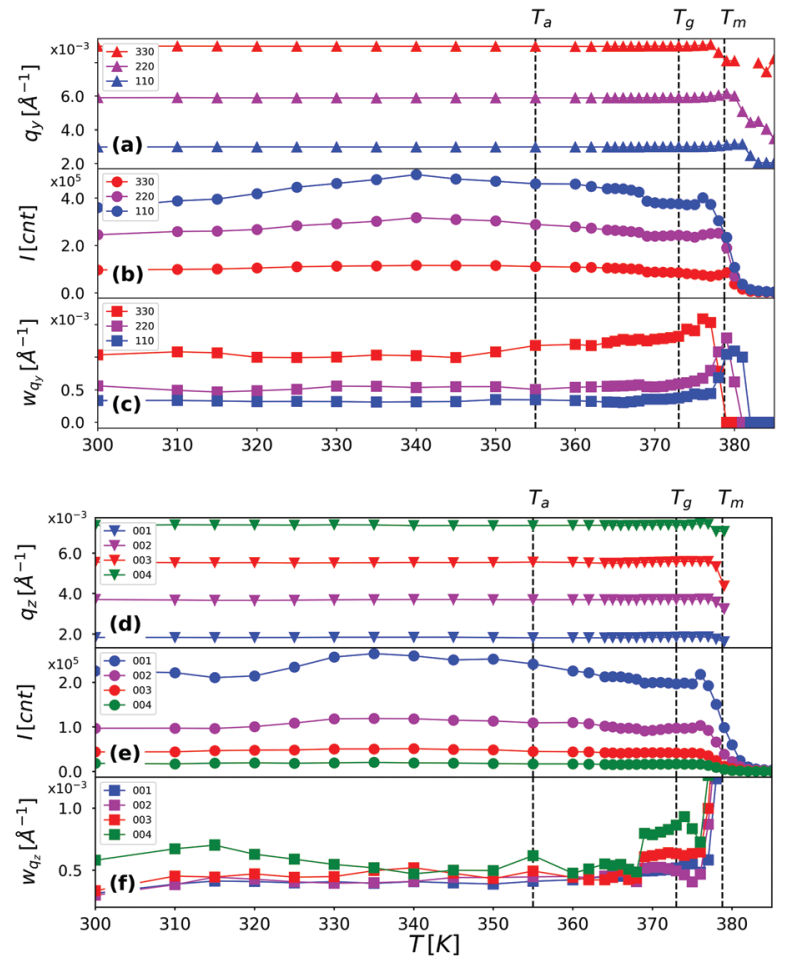

Fig. 5 Temperature dependences of GTSAXS peak positions, integrated peak intensities, and widths of the PS colloidal crystal sample A for the $(a-c)$ in-plane and $(d-f)$ out-of-plane directions.

and out-of-plane directions in a colloidal crystal were probed by choosing the sets of (110) and (001) diffraction orders (Fig. 3). Thus, the obtained temperature dependencies of the peak parameters of sample A are shown in Fig. 5 (results for samples $\mathrm{B}$ and $\mathrm{C}$ are shown in Fig. S2 and S3, ESI $\dagger$ ). At the initial stage of the temperature rise up to $T_{\mathrm{a}}$, the colloidal crystal undergoes thermal expansion while maintaining the long-range order. In this temperature range, both the in-plane and out-of-plane $q$-values of diffraction peaks decrease linearly. By converting $q$-values to interparticle distances, we determined the thermal expansion coefficient of the PS crystal from a linear fit (Fig. 6). The obtained in-plane and out-of-plane linear coefficients $\alpha_{\mathrm{L}}$ of thermal expansion of PS of $6.4 \times 10^{-5} \mathrm{~K}^{-1}$ and $7.2 \times 10^{-5} \mathrm{~K}^{-1}$ are in good agreement with the literature data $^{56}$ reporting the volumetric

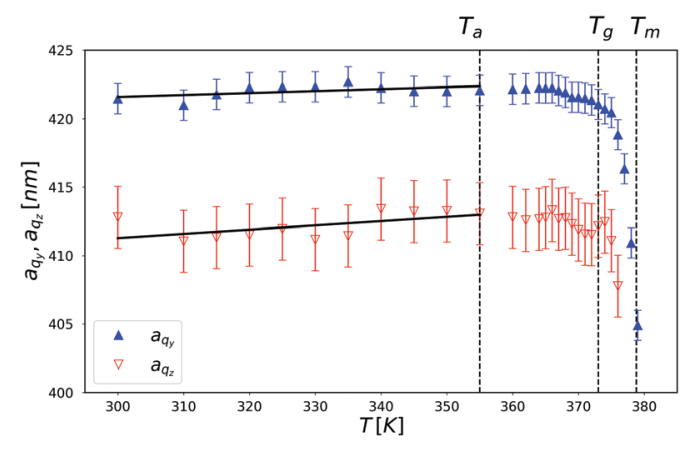

Fig. 6 Temperature dependences of in-plane $a_{q_{y}}(\Delta)$ and out-of-plane $a_{q_{z}}$ $(\nabla)$ interparticle distances for sample A. Linear fits are plotted by solid lines. 
thermal expansion coefficient $\alpha_{\mathrm{V}}=3 \alpha_{\mathrm{L}}$ for PS as $1.7-2.4 \times 10^{-4} \mathrm{~K}^{-1}$. A further temperature increase up to the glass transition temperature of PS, $T_{\mathrm{g}}=373 \mathrm{~K}$, results in a decrease of the particle size due to the faceting of colloidal spheres. In the range from $T_{\mathrm{g}}$ to the melting temperature $T_{\mathrm{m}}$, the particle size decreases more sharply, which is caused by the fusion process of PS particles. From the integrated intensity plot (Fig. 5b and e), we can directly determine the crystal melting temperature $T_{\mathrm{m}}$, i.e. the temperature corresponding to the amorphisation of a colloidal crystal and the formation of a polymer film. $T_{\mathrm{m}}$ values of $378 \mathrm{~K}, 381 \mathrm{~K}$, and $377 \mathrm{~K}$ have been obtained for the samples A, B and C, respectively. The observed increase of $T_{\mathrm{m}}$ with an increase of the film thickness conforms with the results of earlier studies of the glass transition in PS films. ${ }^{57}$ Apart from the $T_{\mathrm{m}}$ behaviour, we observed no significant influence of the PS particle size on the evolution of structural parameters of colloidal crystals in the process of dry sintering.

Temperature dependences of peak widths $W_{q_{y, z}}$ were fitted using the Williamson-Hall (WH) formula: ${ }^{45}$

$$
W_{q_{y, z}}(T)^{2}=\left(2 \pi / L_{q_{y, z}}(T)\right)^{2}+\left(g_{q_{y, z}}(T) q\right)^{2},
$$

where $L_{q_{y, z}}$ are the coherent scattering domain (CSD) sizes and $g_{q_{y, z}}$ represent the lattice deformation parameters. The temperature dependences of CSD sizes and lattice deformation parameters obtained from the fitting analysis are plotted in Fig. 7 for sample A. The CSD size values of $L_{q_{y}}=4 \mu \mathrm{m}$ and $L_{q_{z}}=3 \mu \mathrm{m}$ and the lattice deformation values of $g_{q_{y}}=4 \%$ and $g_{q_{z}}=6 \%$ for the in-plane and out-of-plane directions, respectively, mainly remain constant in the range from RT to the annealing temperature. At $T=365 \mathrm{~K}$, the in-plane CSD size exhibits a peak that coincides with a minimum of the out-of-plane $g$ parameter, indicating the initiation of an intermediate ordered phase in the colloidal crystal due to thermal annealing. An even more pronounced effect of the in-plane CSD size enhancement was observed for sample C (Fig. S4, ESI $\dagger$ ),
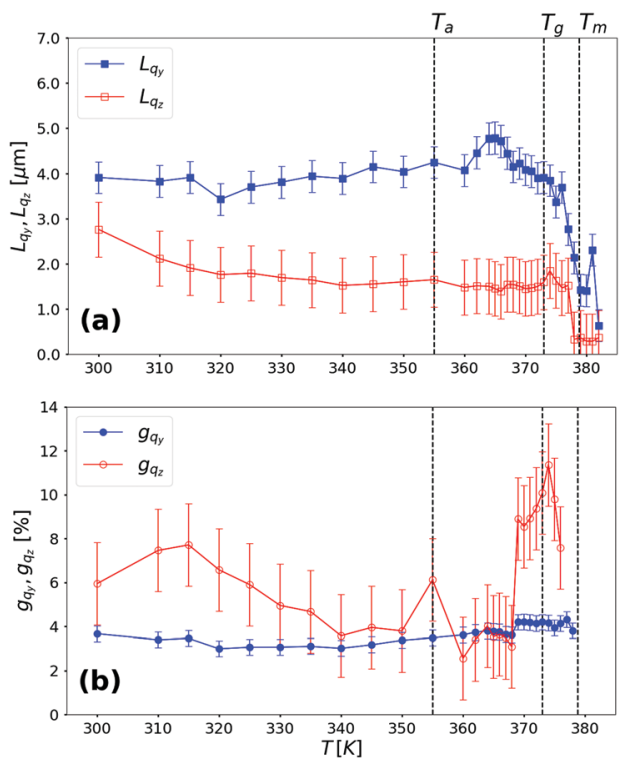

Fig. 7 Temperature dependences of (a) CSD sizes and (b) lattice deformation parameters for the in-plane (blue filled dots) and out-ofplane (red open dots) directions of sample A.
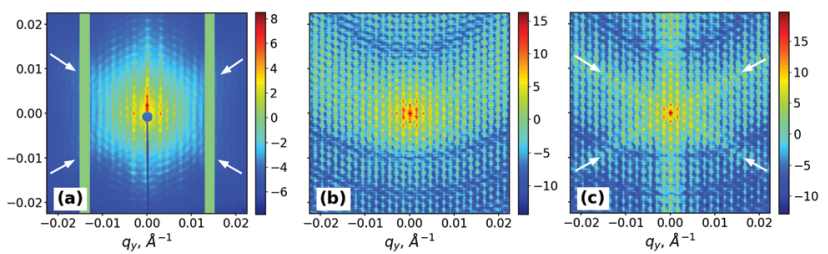

Fig. 8 GTSAXS patterns for sample $A$ at $T=376 \mathrm{~K}$ : (a) experiment, (b) simulation using the scattering function of a single sphere and (c) simulation using the scattering functions of a sphere and a rhombic dodecahedron.

which allows us to conclude that the out-of-plane strain release leads to an enhancement of in-plane ordering in a colloidal crystal due to thermal annealing. With the further raising of temperature up to $T_{g}$, the $L$ values decrease while the $g$ values increase, thus indicating the accommodation of lattice strains due to the fusion of colloidal particles.

At temperatures above $T_{\mathrm{a}}$, the transformation of a spherical particle to a faceted shape sets in, as indicated by an enhancement of the anisotropic diffuse scattering pattern in the form of inclined streaks (depicted by arrows in Fig. 4 and Fig. 8(a, c)). A similar enhancement of diffuse scattering due to particle faceting was observed previously in transmission SAXS geometry. ${ }^{30,45}$ It is of note that the diffuse scattering enhancement observed in the GTSAXS geometry is less pronounced than in the case of the SAXS geometry because of the averaging of the scattering signal in GTSAXS due to a larger beam footprint. We analysed the effect of a particle shape transformation by GTSAXS simulations implementing the scattering function of a rhombic dodecahedron, which can be computed as the sum of the scattering functions of a cube and six equilateral square pyramids attached to the faces of the cube. ${ }^{58}$ In Fig. 8, the GTSAXS pattern measured for the sample A at $T=376 \mathrm{~K}$ is compared to the simulated GTSAXS patterns, corresponding to the scattering function of a single sphere (Fig. $8 \mathrm{~b}$ ) and a 1:1 mixture of spheres and rhombic dodecahedrons (Fig. 8c). As can be deduced from the scattering patterns and corresponding intensity profiles (Fig. S5, ESI $\dagger$ ), the combination of spherical and dodecahedron shapes provides a better description of the experiment as compared to the single sphere case. This result further implies that the shape transformation affects not all particles in a crystal, which is apparently caused by the presence of disordered domains. The transition of PS colloidal particles from spherical to a dodecahedron shape is also indicated by the decrease of lattice spacing at temperatures above $T_{\mathrm{g}}$. As can be seen from Fig. 6, the in-plane lattice spacing decreases from $420 \mathrm{~nm}$ down to $405 \mathrm{~nm}$, reaching the expected value $D_{\mathrm{rd}} \approx 0.96 D_{\mathrm{sp}}$ for the lateral size of a rhombic dodecahedron of the same volume as the initial sphere of diameter $D_{\mathrm{sp}}{ }^{59}$

The structural evolution of a PS colloidal crystal in the process of dry sintering is visualised by a schematic 3D model in Fig. 9, introducing a close-packed fcc arrangement of colloidal spheres with a central sphere surrounded by its 12 neighbouring spheres. The middle layer consisting of the central sphere and its 6 neighbouring spheres (shown in red) is sandwiched between adjacent layers of 3 spheres each (shown in blue and green colours). The thermal expansion stage is followed by particle 


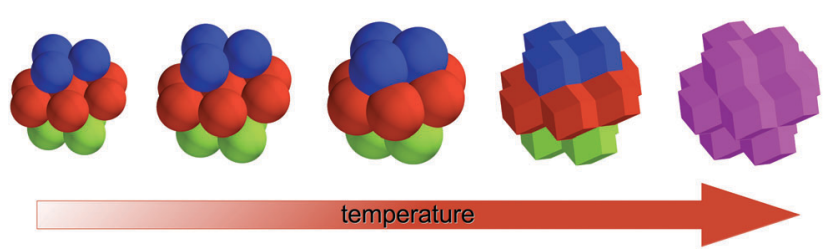

Fig. 9 Schematic 3D model illustrating the structural rearrangement of fcc closed-packed colloidal spheres in the process of dry sintering.

softening, which results in the particle shape transformation from a sphere to a rhombic dodecahedron. The shape transformation is followed by the particle fusion stage, where the boundaries between particles merge together due to the interdiffusion of polymer chains.

\section{Conclusions}

In summary, the structural rearrangement of polymer colloidal crystals in the process of dry sintering has been revealed by in situ GTSAXS measurements, which are sensitive to both in-plane and out-of-plane ordering. By simulations of GTSAXS patterns based on DWBA theory, the structural characteristics such as particle size, number of layers, domain sizes, lattice disorder and mosaic spread have been determined at RT for the colloidal crystals made of spherical PS particles of different sizes. We observed an in-plane lattice dilatation of $1-2 \%$ as compared to the out-of-plane direction in the PS colloidal crystals due to the entropy-driven rhcp arrangement of particle layers and surface tension effects.

We found that the PS colloidal crystals undergo several stages of structural evolution in the process of dry sintering: thermal expansion, particle shape transformation and crystal amorphisation. By analysing the peak positions as a function of temperature, the linear coefficient of thermal expansion of polystyrene was determined, being in excellent agreement with the literature data. At temperatures around the glass transition temperature of PS, we observed a characteristic enhancement of diffuse scattering, indicating a particle transformation from a spherical to polyhedron shape. The observed formation of polyhedral colloidal particles under dry sintering conditions in PS colloidal crystals was supported by GTSAXS simulations using the particle shape functions of a sphere and a rhombic dodecahedron.

At temperatures approaching the crystal melting temperature $T_{\mathrm{m}}$, the softening of colloidal particles and interdiffusion of polymer chains lead to the blurring of interfaces between particles and the subsequent formation of an amorphous polymer film. We observed a linear dependence of $T_{\mathrm{m}}$ on the thickness of the colloidal crystal, in agreement with previous studies of the glass transition in PS films. The observed decay of diffraction peak intensities and scattering domain sizes around $T_{\mathrm{m}}$ implies that the complete loss of ordering and formation of a polymer film occur within a narrow temperature interval of less than $3 \mathrm{~K}$.

Our study enables a novel approach to investigate the structural rearrangement of polymer colloidal crystals under dry sintering conditions. The revealed phase behaviour and intermediate states of colloidal particles in a crystal provide new insights into the self-assembly of soft matter nanostructures with on-demand optical properties.

\section{Conflicts of interest}

There are no conflicts to declare.

\section{Acknowledgements}

The authors thank the whole P10 team and especially M. Kampmann and D. Weschke for their excellent technical support of the experiment. We thank M. Schroer for the careful reading of the manuscript and valuable remarks. The research was carried out at PETRA III at DESY, a member of the Helmholtz Association (HGF).

\section{Notes and references}

1 S. Johnson and J. Joannopoulos, Photonic Crystals: The Road from Theory to Practice, Kluwer Academic Publishers, Norwell, 2002.

2 E. Chow, S. Lin, S. Johnson, P. Villeneuve, J. Joannopoulos, J. Wendt, G. Vawter, W. Zubrzycki, H. Hou and A. Alleman, Nature, 2000, 407, 983-986.

3 T. Gorishnyy, C. K. Ullal, M. Maldovan, G. Fytas and E. L. Thomas, Phys. Rev. Lett., 2005, 94, 115501.

4 R. B. Wehrspohn and J. Üpping, J. Opt., 2012, 14, 024003.

5 F. A. Nutz, P. Ruckdeschel and M. Retsch, J. Colloid Interface Sci., 2015, 457, 96-101.

6 M. Campbell, D. N. Sharp, M. T. Harrison, R. G. Denning and A. J. Turberfield, Nature, 2000, 404, 53-56.

7 G. Subramania and S. Y. Lin, Appl. Phys. Lett., 2004, 85, 5037-5039.

8 M. Stimulak and M. Ravnik, Soft Matter, 2014, 10, 6339-6346.

9 J. Hilhorst, V. V. Abramova, A. Sinitskii, N. A. Sapoletova, K. S. Napolskii, A. A. Eliseev, D. V. Byelov, N. A. Grigoryeva, A. V. Vasilieva, W. G. Bouwman, K. Kvashnina, A. Snigirev, S. V. Grigoriev and A. V. Petukhov, Langmuir, 2009, 25, 10408-10412.

10 J.-M. Meijer, F. Hagemans, L. Rossi, D. V. Byelov, S. I. Castillo, A. Snigirev, I. Snigireva, A. P. Philipse and A. V. Petukhov, Langmuir, 2012, 28, 7631-7638.

11 P. F. Damasceno, M. Engel and S. C. Glotzer, ACS Nano, 2012, 6, 609-614.

12 P. F. Damasceno, M. Engel and S. C. Glotzer, Science, 2012, $337,453-457$.

13 A. V. Petukhov, J.-M. Meijer and G. J. Vroege, Curr. Opin. Colloid Interface Sci., 2015, 20, 272-281.

14 A. V. Petukhov, R. Tuinier and G. J. Vroege, Curr. Opin. Colloid Interface Sci., 2017, 30, 54-61.

15 M. N. O’Brien, H.-X. Lin, M. Girard, M. Olvera de la Cruz and C. A. Mirkin, J. Am. Chem. Soc., 2016, 138, 14562-14565.

16 U. Agarwal and F. A. Escobedo, Nat. Mater., 2011, 10, 230-235. 
17 J.-M. Meijer, A. Pal, S. Ouhajji, H. N. W. Lekkerkerker, A. P. Philipse and A. V. Petukhov, Nat. Commun., 2017, 8, 14352.

18 J. Gong, R. S. Newman, M. Engel, M. Zhao, F. Bian, S. C. Glotzer and Z. Tang, Nat. Commun., 2017, 8, 14038.

19 H. Míguez, F. Meseguer, C. López, Á. Blanco, J. S. Moya, J. Requena, A. Mifsud and V. Fornés, Adv. Mater., 1998, 10, 480-483.

20 B. Gates, S. H. Park and Y. Xia, Adv. Mater., 2000, 12, 653-656.

21 S. Kuai, Y. Zhang, V.-V. Truong and X. Hu, Appl. Phys. A: Mater. Sci. Process., 2002, 74, 89-90.

22 X. Liu, D. Zhao, C. Geng, L. Zhang, T. Tan, M. Hu and Q. Yan, J. Colloid Interface Sci., 2014, 434, 98-103.

23 F. A. Nutz and M. Retsch, Phys. Chem. Chem. Phys., 2017, 19, 16124-16130.

24 H. R. Vutukuri, A. Imhof and A. van Blaaderen, Angew. Chem., Int. Ed., 2014, 53, 13830-13834.

25 C. X. Du, G. van Anders, R. S. Newman and S. C. Glotzer, Proc. Natl. Acad. Sci. U. S. A., 2017, 114, E3892-E3899.

26 N. Tian, Z.-Y. Zhou, S.-G. Sun, Y. Ding and Z. L. Wang, Science, 2007, 316, 732-735.

27 N. Becknell, Y. Son, D. Kim, D. Li, Y. Yu, Z. Niu, T. Lei, B. T. Sneed, K. L. More, N. M. Markovic, V. R. Stamenkovic and P. Yang, J. Am. Chem. Soc., 2017, 139, 11678-11681.

28 P. Schall, I. Cohen, D. A. Weitz and F. Spaepen, Nature, 2006, 440, 319-323.

29 B. Hatton, L. Mishchenko, S. Davis, K. H. Sandhage and J. Aizenberg, Proc. Natl. Acad. Sci. U. S. A., 2010, 107, 10354-10359.

30 A. V. Zozulya, J.-M. Meijer, A. Shabalin, A. Ricci, F. Westermeier, R. P. Kurta, U. Lorenz, A. Singer, O. Yefanov, A. V. Petukhov, M. Sprung and I. A. Vartanyants, J. Appl. Crystallogr., 2013, 46, 903-907.

31 M. A. Schroer, F. Westermeier, F. Lehmkühler, H. Conrad, A. Schavkan, A. V. Zozulya, B. Fischer, W. Roseker, M. Sprung, C. Gutt and G. Grübel, J. Chem. Phys., 2016, 144, 084903.

32 B. M. Murphy, S. Festersen and O. M. Magnussen, Nanoscale, 2016, 8, 13859-13866.

33 A. V. Petukhov, I. P. Dolbnya, D. G. A. L. Aarts, G. J. Vroege and H. N. W. Lekkerkerker, Phys. Rev. Lett., 2003, 90, 028304.

34 A. V. Petukhov, J. H. J. Thijssen, D. C. 't Hart, A. Imhof, A. van Blaaderen, I. P. Dolbnya, A. Snigirev, A. Moussad and I. Snigireva, J. Appl. Crystallogr., 2006, 39, 137-144.

35 J. Thijssen, A. Petukhov, D. 't Hart, A. Imhof, C. van der Werf, R. Schropp and A. van Blaaderen, Adv. Mater., 2006, 18, 1662-1666.

36 S. V. Roth, P. Müller-Buschbaum, A. Timmann, J. Perlich and R. Gehrke, J. Appl. Crystallogr., 2007, 40, s346-s349.

37 P. Huber, O. Bunk, U. Pietsch, M. Textor and T. Geue, J. Phys. Chem. B, 2010, 114, 12473-12479.

38 J. Gulden, O. M. Yefanov, A. P. Mancuso, V. V. Abramova, J. Hilhorst, D. Byelov, I. Snigireva, A. Snigirev, A. V. Petukhov and I. A. Vartanyants, Phys. Rev. B: Condens. Matter Mater. Phys., 2010, 81, 224105.

39 A. G. Shabalin, J.-M. Meijer, R. Dronyak, O. M. Yefanov, A. Singer, R. P. Kurta, U. Lorenz, O. Y. Gorobtsov, D. Dzhigaev, S. Kalbfleisch,
J. Gulden, A. V. Zozulya, M. Sprung, A. V. Petukhov and I. A. Vartanyants, Phys. Rev. Lett., 2016, 117, 138002.

40 S. Lazarev, I. Besedin, A. V. Zozulya, J.-M. Meijer, D. Dzhigaev, O. Y. Gorobtsov, R. P. Kurta, M. Rose, A. G. Shabalin, E. A. Sulyanova, I. A. Zaluzhnyy, A. P. Menushenkov, M. Sprung, A. V. Petukhov and I. A. Vartanyants, Small, 2018, 14, 1702575.

41 S. Hu, J. Rieger, S. V. Roth, R. Gehrke, R. J. Leyrer and Y. Men, Langmuir, 2009, 25, 4230-4234.

42 S. Hu, J. Rieger, Z. Yi, J. Zhang, X. Chen, S. V. Roth, R. Gehrke and Y. Men, Langmuir, 2010, 26, 13216-13220.

43 X. Chen, S. Fischer, Z. Yi, V. Boyko, A. Terrenoire, F. Reinhold, J. Rieger, X. Li and Y. Men, Langmuir, 2011, 27, 8458-8463.

44 G. Herzog, M. M. Abul Kashem, G. Benecke, A. Buffet, R. Gehrke, J. Perlich, M. Schwartzkopf, V. Körstgens, R. Meier, M. A. Niedermeier, M. Rawolle, M. A. Ruderer, P. Müller-Buschbaum, W. Wurth and S. V. Roth, Langmuir, 2012, 28, 8230-8237.

45 E. A. Sulyanova, A. Shabalin, A. V. Zozulya, J.-M. Meijer, D. Dzhigaev, O. Gorobtsov, R. P. Kurta, S. Lazarev, U. Lorenz, A. Singer, O. Yefanov, I. Zaluzhnyy, I. Besedin, M. Sprung, A. V. Petukhov and I. A. Vartanyants, Langmuir, 2015, 31, 5274-5283.

46 G. Renaud, R. Lazzari and F. Leroy, Surf. Sci. Rep., 2009, 64, 255-380.

47 A. Hexemer and P. Müller-Buschbaum, IUCrJ, 2015, 2, 106-125.

48 X. Lu, K. G. Yager, D. Johnston, C. T. Black and B. M. Ocko, J. Appl. Crystallogr., 2013, 46, 165-172.

49 N. Mahadevapuram, J. Strzalka and G. E. Stein, J. Polym. Sci., Part B: Polym. Phys., 2013, 51, 602-610.

50 S. K. Sinha, E. B. Sirota, S. Garoff and H. B. Stanley, Phys. Rev. B: Condens. Matter Mater. Phys., 1988, 38, 2297-2311.

51 M. Rauscher, T. Salditt and H. Spohn, Phys. Rev. B: Condens. Matter Mater. Phys., 1995, 52, 16855-16863.

52 B. Lee, I. Park, J. Yoon, S. Park, J. Kim, K.-W. Kim, T. Chang and M. Ree, Macromolecules, 2005, 38, 4311-4323.

53 M. P. Tate, V. N. Urade, J. D. Kowalski, T.-c. Wei, B. D. Hamilton, B. W. Eggiman and H. W. Hillhouse, J. Phys. Chem. B, 2006, 110, 9882-9892.

54 I. A. Vartanyants, D. Grigoriev and A. V. Zozulya, Thin Solid Films, 2007, 515, 5546-5552.

55 J.-M. Meijer, A. Shabalin, R. Dronyak, O. M. Yefanov, A. Singer, R. P. Kurta, U. Lorenz, O. Gorobstov, D. Dzhigaev, J. Gulden, D. V. Byelov, A. V. Zozulya, M. Sprung, I. A. Vartanyants and A. V. Petukhov, J. Appl. Crystallogr., 2014, 47, 1199-1204.

56 G. Beaucage, R. Composto and R. Stein, J. Polym. Sci., Part B: Polym. Phys., 1993, 31, 319-326.

57 J. L. Keddie, R. A. L. Jones and R. A. Cory, EPL, 1994, 27, 59. 58 A. Senesi and B. Lee, J. Appl. Crystallogr., 2015, 48, 565-577. 59 The volume of a sphere $V_{\mathrm{sp}}=(4 / 3) \pi R_{\mathrm{sp}}{ }^{3}$ has to be equal to the volume of a rhombic dodecahedron $V_{\mathrm{rd}}=(16 \sqrt{3} / 9) a^{3}$, where the radius of a sphere $R_{\mathrm{sp}}=0.5 D_{\mathrm{sp}}$ and $a$ is the edge of a rhombic dodecahedron. From this condition, the lateral size of a rhombic dodecahedron $D_{\mathrm{rd}}=\sqrt{3} a \approx 0.96 D_{\mathrm{sp}}$. 